\title{
ACMSA: Antarctic Centre for Millimetre and Sub-millimetre Astrophysics
}

\author{
Giorgio Sironi \\ Physics Dept. - University of Milano Bicocca - Milano, Italy
}

\begin{abstract}
.
A Working Group has been created to study the feasibility of ACMSA: Antarctic Center for Millimetric and Sub-millimetric Astrophysics, an international facility based at Dome Concordia (Dome C).
\end{abstract}

Tests made over the last ten years show that the Antarctic Plateau, an elevated and extended area, characterized by thin, dry and stable atmosphere, is the most convenient place for ground based observations of the sky at mm and sub-mm wavelengths. Better conditions can be found only in space.

Facilities for mm- sub-mm astronomy on the Antarctic Plateau have been so far available only at the Amundsen Scott base at South Pole, but are insufficient to satisfy the international community needs. To complement South Pole, a group of astrophysicists from Europe, Australia and USA (Olmi 2004) recently suggested the creation of a new $\mathrm{mm}$ and sub-mm facility at Dome C, ACMSA (the Antarctic Centre for Millimetric and Sub-millimetric Astrophysics). Here the expected observing conditions are even better that at South Pole. The French - Italian Dome Concordia base will soon become operational all year round.

To prepare a proposal for ACMSA a Working Group (WG) has been formed. The aim is a scientific and technical case which will: i) investigate how selected astrophysical and cosmological questions can be converted into coherent frontier science at sub-mm wavelengths, ii) propose facilities and experiments, iii) review existing facilities and experiments on the Antarctic Plateau and elsewhere, iv) study the state of the art technology available and the use of robotized systems and v)make cost evaluations. The WG will: a) inform the scientific community at large, b) look for collaborations, c) search for links with national and international organizations (French - Italian Concordia Program, European Polar Board, SCAR, IAU Antarctic Working Group, ESO, NSF etc.)

\section{References}

Olmi, L. 2004, Memorie della Societa Astronomica Italiana Supplement, 5, 368 